\title{
Register of Retinoblastoma: Preliminary Results
}

\author{
MARCELLE JAY, JOHN COWELL and JOHN HUNGERFORD \\ London
}

The epidemiology and genetics of retinoblastoma $(\mathrm{Rb})$ are well documented and have been described from pooled studies and personal observations by Vogel. ${ }^{1}$ Large series of cases with $\mathrm{Rb}$ have been studied in France ${ }^{2}$ and in the Netherlands, ${ }^{3}$ and the present study concerns the preliminary results derived from a register of $\mathrm{Rb}$ cases of all ages born in the United Kingdom. The aim of the register is to ascertain all cases of Rb born since 1940, so that it will be possible to derive reliable figures for genetic counselling, and to study the molecular biology of $\mathbf{R b}$.

\section{Material and Methods}

Patients with $\mathrm{Rb}$ are ascertained from various sources, these include the series of patients treated or seen at Moorfields Eye Hospital and at St Bartholomew's Hospital, cancer registries, and individual ophthalmologists, pathologists and paediatric oncologists with an interest in $\mathrm{Rb}$ who are willing to send details of their cases for inclusion in the register.

The details included in the register and stored on computer are: name, date of birth, hospital number, laterality and presence or absence of family history. These data represent the minimum required, and additional data included whether a twin birth, sibship size and rank, age at diagnosis of tumour in first and second eye, and parental age. The paternal age is of particular interest, as a raised paternal age is found in a number of autosomal dominant disorders where there are new mutations. ${ }^{4}$

There are 19 cancer registries in the United Kingdom, and they will release details, including the identity of the patient, providing suitable ethical precautions concerning the confidentiality of records are observed. Cancer registries will also release the name of the consultant ophthalmologist and of the family practitioner, so that further details of family history may be obtained when relevant.

\section{Results}

The preliminary results are based on 1,302 cases of $\mathrm{Rb}$ of all ages which are drawn from 1,076 families.

\section{Ascertainment}

The distribution of cases born since 1945 is shown in the Figure. The unexpected peak of 50 cases born in 1955, which was not a year with a record number of births, is used to indicate a possible maximum number of new cases born per annum. If the estimate of new cases per annum is somewhere between 40 and 50 , then ascertainment so far has reached about 60 per cent of all cases. These have to be estimates, since an unknown number of cases with late onset, between the ages of 3 and 10 years, have to be allowed for, and therefore precise prevalence figures have to be retrospective.

The incidence of retinoblastoma may be rising as a result of an increasing number of individuals with the hereditary form of the disease surviving to reproductive age. Total ascertainment over a period of two generations is required in order to compare the incidence per 10,000 births in each generation.

\section{Hereditary Retinoblastoma}

Cases of $\mathrm{Rb}$ may be hereditary or non-hereditary. All bilateral, and 15 per cent of unilateral, cases of $\mathrm{Rb}$ are hereditary, and hereditary $\mathbf{R b}$ is transmitted as an autosomal dominant trait from an affected parent to an affected child. This usually occurs from generation to generation in an unbroken line, 


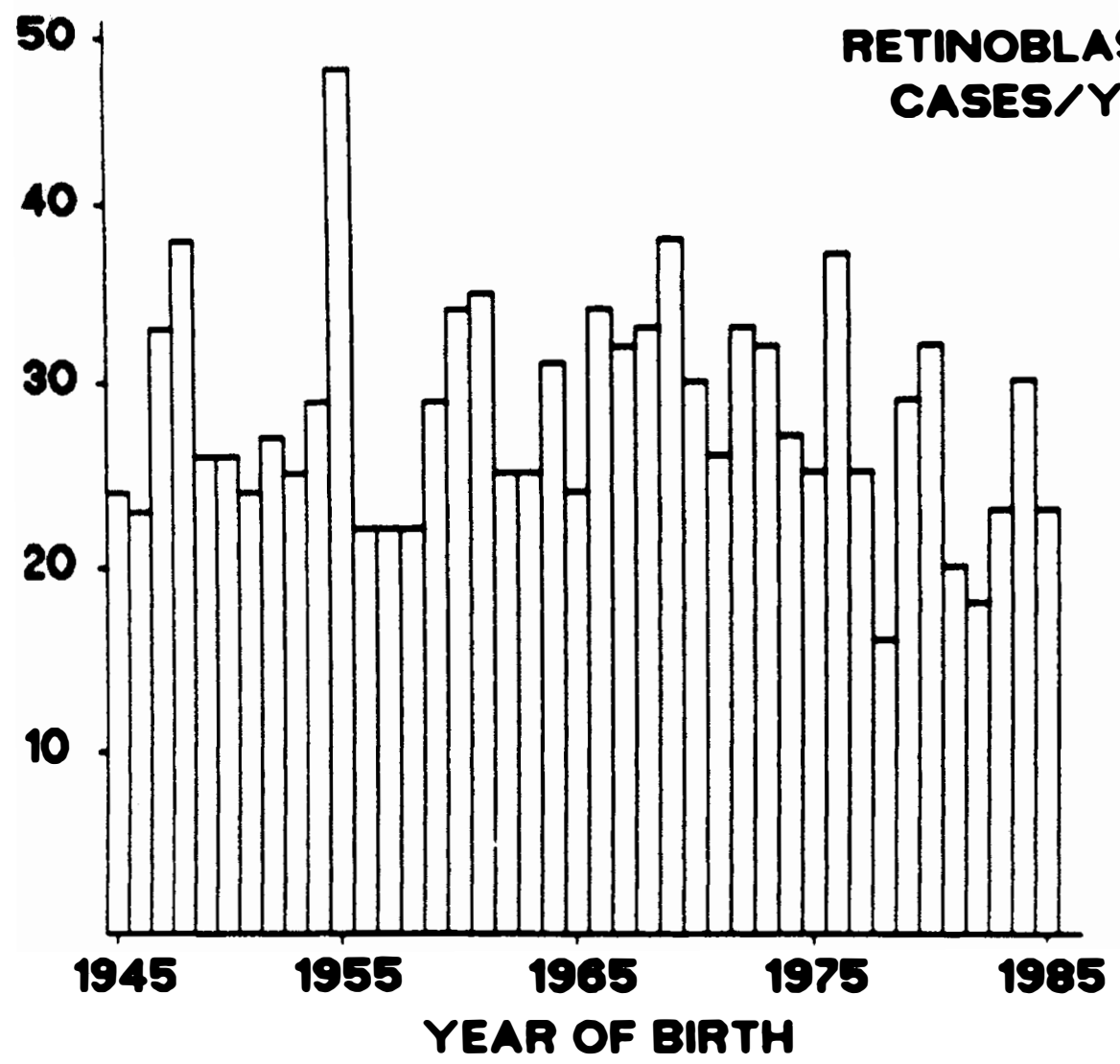

Fig. Number of cases of retinoblastoma born each year between 1945 and 1985 .

although there are examples, albeit rare in the present series, of an unaffected individual with an affected parent and an affected child. This is an example of non-penetrance which is said to occur in 10 per cent of families. ${ }^{1}$ Each individual affected with hereditary retinoblastoma has a 50 per cent risk of having an affected child. The risk in practice is very slightly less than this, to allow for those rare individuals showing non-penetrance.

There are 1,076 index patients (one index patient for each family) in the present series. The hereditary proportion consists of all bilateral cases, and all unilateral cases with a positive family history, which in this series is 47 per cent. It is interesting that this result is at variance with the hereditary proportion of 40 per cent quoted in the literature. ${ }^{1}$ The present series is by no means complete, and it is expected that the proportion of bilateral cases will go down as ascertainment approaches 100 per cent.

Bilateral $\mathrm{Rb}$ forms the largest contribution to the total of hereditary cases, and some bilateral $\mathrm{Rb}$ have a positive family history. Our results so far show a somewhat higher proportion ( 22 per cent) of familial cases of bilateral $\mathrm{Rb}$ than expected, and this may reflect a bias of referral. Lists of cases of $R b$ received from centres specialising in the treatment of $\mathrm{Rb}$ show similar biases, a large proportion of bilateral cases, and a large number of cases with a positive family history.

Unilateral $\mathbf{R b}$ may also be hereditary. It is obviously hereditary when there is a positive family history, and it is also hereditary in those cases where there are multifocal tumours in the affected eye. The proportion of hereditary 
unilateral $\mathrm{Rb}$ is said to be 15 per cent, ${ }^{1}$ whereas in the present series it is only 4 per cent. The much higher proportion in the literature may be due to a tendency to publish cases of unilateral $\mathrm{Rb}$ that have a positive family history. In the present series, there are 5 pedigrees where all affected individuals have unilateral $\mathrm{Rb}$, and 9 pedigrees where a parent with unilateral $\mathrm{Rb}$ has had a child with bilateral disease. These interesting pedigrees can be explained by the 'two-hit' hypothesis of Knudson ${ }^{5}$ which states that hereditary retinoblastoma is the result of a germinal mutation followed by a somatic mutation.

\section{Chromosome deletions}

The last subgroup of hereditary $\mathrm{Rb}$ consists of those cases with a chromosome deletion. Deletions of a small segment of the long arm of chromosome 13 are found in bilateral and unilateral $\mathrm{Rb}$ and the proportion is said to be between 5 and 10 per cent, ${ }^{1}$ although preliminary data suggest this figure may be too high. Chromosome deletions in Rb are likely to be over-reported and we have found 18 deletions in the first 400 consecutive cases in the St Bartholomew's/Moorfields series, a proportion of 5 per cent. This proportion has been found in patients drawn from all over the United Kingdom, and it has remained constant with time. ${ }^{6}$

The deletions found span various regions of the long arm of chromosome 13 , and the portion which is always deleted lies in band q14 of chromosome 13 . There is reason to believe that hereditary cases of retinoblastoma are due to a deletion, perhaps of a promoter sequence of a cancer suppressing gene which operates during fetal development and resulting in early onset. These deletions may not always be visible microscopically, but may be demonstrated at the level of the enzyme, esterase $\mathrm{D}$, whose locus lies close to that of the retinoblastoma predisposing gene. When there is a deletion which includes the locus of esterase $D$, the level of this enzyme in red blood cells is 50 per cent of the normal, and it is possible to think of the first, and germinal mutation of the Knudson hypothesis in terms of a deletion. The recent cloning of a candidate for the retinoblastoma gene ${ }^{7}$ may identify submicroscopic deletions.
Individuals carrying a large deletion usually have characteristic congenital abnormalities which are sufficient to indicate the presence of a deletion. Smaller deletion carriers however may have no other phenotypic consequences and the subtle changes involved may not be detected using conventional chromosome banding techniques. We have used esterase D quantitation to identify some of these patients unequivocally. Up to the present we have found 6 patients who do not have any clinical evidence of a deletion, but who have low enzyme levels, thus emphasising the importance of this test which is provided on a routine basis at the Institute of Child Health. Esterase D can also be used for prenatal testing, ${ }^{8}$ but DNA probes will prove more useful because of the greater possibility of natural variation.

\section{Non-hereditary retinoblastoma}

Non-hereditary retinoblastoma is the result of two somatic mutations affecting the same target cell, an embryonic retinoblast. In the present series, non-hereditary cases appear to comprise over 90 per cent of all unilateral $\mathrm{Rb}$. Clinically, and even pathologically, it is often impossible to distinguish between a single, unifocal tumour, and multifocal tumours which have coalesced and resemble a single tumour.

As a consequence, the risk figure for the offspring of individuals with unilateral $\mathrm{Rb}$ has been calculated empirically. ${ }^{1}$ If under 10 per cent of unilateral retinoblastomas are hereditary because of a positive family history, and those who are hereditary have a 50 per cent risk of having an affected child, the figure arrived at is the product of the two, namely 5 per cent or less. It must be stressed that this is an empirical risk derived from incomplete studies, and it is still too early to calculate a precise risk from the present study.

\section{Conclusions}

While the present study is in its early stages, and it is not possible to derive accurate statistics of prevalence, or to do more than to identify certain trends. It is possible however to estimate that there are probably 600 individuals with $\mathrm{Rb}$ and of child bearing age, and it is likely that a large number of them will be 
seeking genetic counselling in the near future due to increased public awareness. It is hoped that large and ongoing studies such as these will provide more accurate data for genetic counselling and for prevalence and genetic studies.

We thank Moorfields Eye Hospital for their support (Locally Organised Clinical Research grants 85/4 and $88 / 1)$. We would also like to thank all the cancer registries and consultant ophthalmologists who have contributed their cases to this study.

\section{References}

1 Vogel F: Genetics of retinoblastoma. Hum Genet 1979, 52: 1-54.

${ }^{2}$ Briard-Guillemot ML, Bonaiti-Pellié C, Feingold J, Frezal J: Etude génétique du retinoblastome. Humangenetik 1974, 24: 271-84.
${ }^{3}$ Schappert-Kimmijser J, Hemmes GD, Nijland R: The heredity of retinoblastoma. Ophthalmologica 1966, 151: 197-213.

${ }^{4}$ Penrose LS: Parental age and mutation. Lancet 1955 , ii: $312-3$.

${ }^{5}$ Knudson AG Jr: Mutation and cancer: statistical study of retinoblastoma. Proc Natl Acad Sci USA 1971, 68: 820-3.

${ }^{6}$ Cowell JK, Rutland P, Jay M, Hungerford J: Deletions of the esterase D locus from a survey of 200 retinoblastoma patients. Hum Genet 1986, 72: 164-7.

${ }^{7}$ Friend SH, Bernards R, Rogelj S, Weinberg RA, Rapaport JM, Albert DM, Dryja TP: A human DNA segment with properties of the gene that predisposes to retinoblastoma and osteosarcoma. Nature 1986, 323: 643-6.

${ }^{8}$ Cowell J, Jay M, Rutland P, Hungerford J: An assessment of the usefulness of electrophoretic variants of esterase $\mathrm{D}$ in the antenatal diagnosis of retinoblastoma in the UK. Br J Cancer 1987, 55: $661-4$ 